\title{
Structure and Dynamics of Liquid Ethanol
}

\author{
L. Saiz and J. A. Padró* \\ Departament de Física Fonamental, Universitat de Barcelona, Diagonal, 647, E-08028 Barcelona, Spain
}

\section{E. Guàrdia}

Departament de Física i Enginyeria Nuclear, Universitat Politècnica de Catalunya, Sor Eulàlia D'Anzizu, Campus Nord, Mòdul B4-B5, E-08034 Barcelona, Spain

Received: June 17, 1996; In Final Form: October 16, $1996^{\otimes}$

\begin{abstract}
Molecular dynamics simulations of liquid ethanol at four thermodynamic states ranging from $T=173 \mathrm{~K}$ to $T=348 \mathrm{~K}$ were carried out using the transferable OPLS potential model of Jorgensen (J. Phys. Chem. 1986, $90,1276)$. Both static and dynamic properties are analyzed. The resulting properties show an overall agreement with available experimental data. Special attention is paid to the hydrogen bonds and to their influence on the molecular behavior. Results for liquid ethanol are compared with those for methanol in earlier computer simulation studies.
\end{abstract}

\section{Introduction}

A deep understanding of the microscopic behavior of $\mathrm{H}$ bonded liquids is indispensable for many chemical and biological processes. However, the influence of $\mathrm{H}$-bonds on the static and dynamic properties of associated liquids is not well understood. Whereas liquid water has been the subject of a large body of experimental and computer simulation studies, less is known of other H-bonded liquids such as alcohols (for a recent review see ref 1). Alcohols with one hydroxyl group are characterized by H-bonding patterns which may be largely regarded as linear chains. This is very different from the tridimensional network typical of water.

One of the simplest alcohols with one hydroxyl group is ethanol, a well-known solvent widely used in the chemical industry. Yet, whereas Monte Carlo (MC) and molecular dynamics (MD) simulations have been carried out for liquid methanol, ${ }^{2-11}$ computer simulations of the seemingly liquid ethanol are rather scarce and restricted to the equilibrium properties. Jorgensen carried out MC simulations of the thermodynamic and structural properties of liquid ethanol at $T$ $=298 \mathrm{~K}$. He used two similar models for the intermolecular interactions, the transferable intermolecular potential (TIPS) ${ }^{12}$ and the optimized potential model for liquid simulations (OPLS), ${ }^{2}$ where the latter is regarded as an improvement on TIPS. The OPLS are a set of transferable effective intermolecular potentials developed by Jorgensen and co-workers for different organic fluids. For the liquid alcohols the TIPS and the OPLS potentials have the same functional form but differ slightly in the values of their parameters.

The MC simulations ${ }^{2}$ show that the OPLS potentials produce thermodynamic and structural results for several liquid alcohols at room temperature which are in good agreement with experiments. Recent Gibbs-ensemble MC calculations, ${ }^{13}$ also using the OPLS potentials, gives a the vapor-liquid coexistence curve for the low alcohols-including ethanol-which is in satisfactory agreement with experiment. Polarizable potentials for liquid alcohols have been recently proposed ${ }^{14,15}$ and used for the simulation of the thermodynamic properties and structure of liquid ethanol. ${ }^{14}$ The results do not show significant differences with those obtained by using OPLS. Although

\footnotetext{
${ }^{\otimes}$ Abstract published in Advance ACS Abstracts, December 1, 1996.
}

TABLE 1: Temperatures and Densities of the Simulated Systems

\begin{tabular}{lcc}
\hline run & temp $(\mathrm{K})$ & density $\left(\mathrm{g} / \mathrm{cm}^{3}\right)$ \\
\hline A & 173 & 0.8936 \\
B & 223 & 0.8492 \\
C & 298 & 0.7873 \\
D & 348 & 0.7401
\end{tabular}

polarizable potentials are admittedly more realistic the substantial increase in computational cost yield, in principle, barely a reasonable dividend. Hence, in this study we have decided to use the pairwise OPLS model.

In this paper we report the results of a MD study of liquid ethanol at four different states, with temperatures ranging from 173 to $348 \mathrm{~K}$. We assume the same OPLS intermolecular potential which was fitted to describe the liquid alcohols at ambient conditions. ${ }^{2}$ The MD simulations reported below include thermodynamic and structural properties as well as transport properties. To our knowledge earlier studies were restricted to static properties at $298 \mathrm{~K}^{2,12,14}$ and higher temperatures. ${ }^{13}$ Computational details of the MD simulations are given in section II. Section III is devoted to the static properties at different temperatures including the analysis of thermodynamic properties, molecular conformation, X-ray and neutron scattering patterns at $298 \mathrm{~K}$, radial distribution functions and orientational order, percentages of molecules in different H-bonding states, and characteristics of H-bonded chains. The transport properties are considered in section IV. This section includes self-diffusion coefficients, velocity autocorrelation functions, and corresponding power spectra as well as reorientational time correlation functions. Finally, in section $\mathrm{V}$, we briefly sum up a few concluding remarks.

\section{Computer Simulation Details}

We carried out MD simulations of liquid ethanol at the four thermodynamic states detailed in Table 1 . We use as input the appropriate experimental temperatures and densities at normal pressure. The simulated system was made up of 125 semiflexible ethanol molecules in a cubic box with periodic boundary conditions. The simulation technique was the same employed in earlier MD studies of liquid methanol., ${ }^{9,11}$ Properties were calculated from MD runs of $10^{5}$ time steps which correspond 
TABLE 2: Intermolecular Potential Energies and Heats of Vaporization (in $\mathrm{kcal} / \mathrm{mol}$ )

\begin{tabular}{lcclc}
\hline & \multicolumn{4}{c}{$T(\mathrm{~K})$} \\
\cline { 2 - 5 } & 173 & 223 & \multicolumn{1}{c}{298} & 348 \\
\hline$-E_{i}(1)$ & 11.28 & 10.76 & 9.69 & 8.78 \\
$\Delta H_{\text {vap }}{ }^{a}$ & 11.60 & 11.20 & 10.23 & 9.27 \\
$\Delta H_{\text {vap }}^{\text {exp }}$ & & 10.79 & 10.15 & 9.37 \\
$\Delta H_{\text {vap }}{ }^{c}$ & & & $9.99,{ }^{d} 10.08^{e}$ & $9.08^{f}$
\end{tabular}

${ }^{a}$ The $\left(H-H^{\circ}\right)$ corrections are not included in $\Delta H_{\text {vap }}$ at 173 and 223 K. ${ }^{b}$ Experimental values from ref $17 \mathrm{~b} .{ }^{c}$ Results from other simulations: ${ }^{d}$ from ref $2 ;{ }^{e}$ from ref $14 ;{ }^{f}$ from ref 13 .

to a time interval of $250 \mathrm{ps}$. We have found it useful to compare the results for ethanol reported in this work with those we obtained for methanol at $T=298 \mathrm{~K} .{ }^{9,11}$

We adopted the OPLS potential and the molecular model proposed by Jorgensen in ref 2. Each ethanol molecule is represented by four interaction sites corresponding to the two methyl groups and the oxygen and hydrogen atoms of the hydroxyl group. Hydrogen atoms of the methyl groups are not explicitly considered. The group close to the $\mathrm{O}$ atom $\left(\mathrm{CH}_{2}\right)$ is termed $\mathrm{Me}_{\mathrm{O}}$, whereas the one at the end of the molecule $\left(\mathrm{CH}_{3}\right)$ is termed $\mathrm{Me}_{\mathrm{C}}$. The bond lengths and the bond $\mathrm{COH}$ and $\mathrm{CCO}$ angles are kept constant, and the only intramolecular motions considered in the simulations are the rotations around the $\mathrm{CO}$ bond. Interactions between sites are described by Lennard-Jones and Coulomb terms, and we note that the $\mathrm{CH}_{3}$ site has no electric charge. The values of the geometrical parameters of molecules, intermolecular and internal potential functions and potential parameters are those of ref 2 .

Lennard-Jones interactions were truncated at half the box length, and the Ewald summation was used for Coulomb interactions. Although the best method to deal with long-range interactions is still under discussion, recent studies on aqueous solutions have shown that the Ewald method leads to more desirable results than other techniques like simple truncation cutoff or switching functions. ${ }^{16}$

\section{Equilibrium Properties}

A. Thermodynamics. The intermolecular potential energy $\left.E_{\mathrm{i}}(1)\right)$ of liquid ethanol at different temperatures was determined during the MD simulations and the results are presented in Table 2. The heat of vaporization was calculated by using

$$
\Delta H_{\mathrm{vap}}=E_{\mathrm{intra}}(\mathrm{g})-E_{\mathrm{i}}(\mathrm{l})-E_{\mathrm{intra}}(\mathrm{l})+R T-\left(H^{\circ}-H\right)
$$

where $E_{\text {intra }}(\mathrm{g})$ and $E_{\text {intra }}(1)$ are the intramolecular torsional energies for gas and liquid, respectively, and $\left(H^{\circ}-H\right)$ is the enthalpy difference between the real and ideal gas. $E_{\text {intra }}(1)$ was obtained from MD simulations and $E_{\text {intra }}(\mathrm{g})$ was computed according to a Boltzmann distribution for the torsional potential function. The values of $\left(H^{\circ}-H\right)$ for $T=298 \mathrm{~K}$ and $T=348$ $\mathrm{K}$ were taken from ref $17 \mathrm{a}$. We do not know the experimental $\left(H^{\circ}-H\right)$ values at the other two temperatures.

Our MD results for $\Delta H_{\text {vap }}$ are in overall agreement with experiment $^{17 \mathrm{~b}}$ (Table 2), though those that may be directly compared with experimental data (for $T=298 \mathrm{~K}$ and $T=348$ $\mathrm{K})$ are approximately $1 \%$ higher. The results of the isothermalisobaric MC simulations of Jorgensen ${ }^{2}$ at room temperature are approximately $1.5 \%$ lower. Moreover, he used cutoff truncation for the intermolecular interactions and a value for the density $4.7 \%$ lower than experiment. We also list in Table 2 two other simulation results for $\Delta H_{\text {vap }}$. One uses a polarizable model and spherical cutoff at $298 \mathrm{~K} ;{ }^{14}$ the other, at $350 \mathrm{~K}$, uses the OPLS
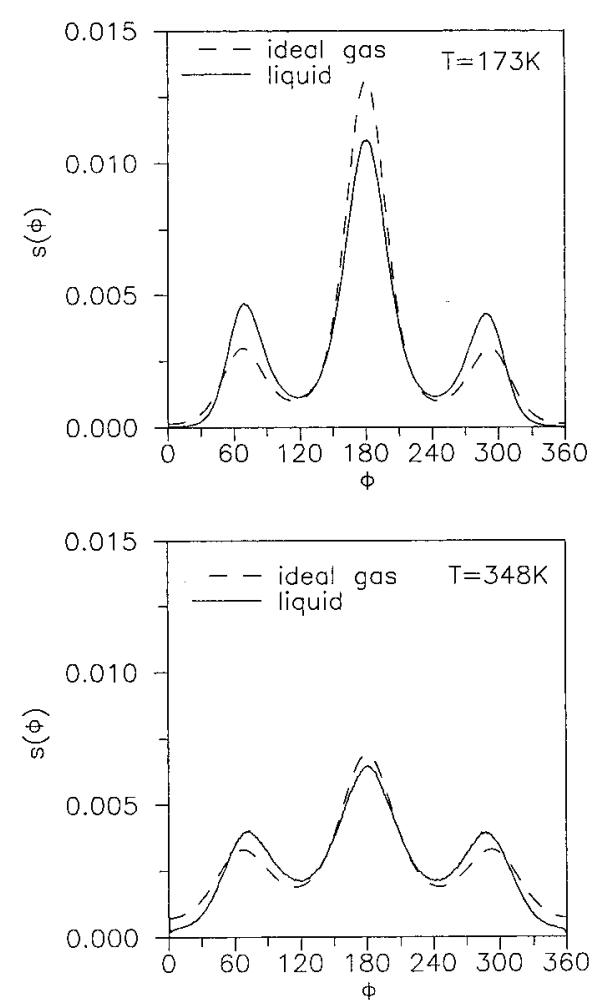

Figure 1. Probability distributions of the torsional dihedral angle around the $\mathrm{C}-\mathrm{O}$ bond.

TABLE 3: Percentages of Gauche Conformers in Liquid and Gas Phases

\begin{tabular}{lcccc}
\hline & \multicolumn{4}{c}{$T(\mathrm{~K})$} \\
\cline { 2 - 5 } & 173 & 223 & 298 & 348 \\
\hline liquid & 41.7 & 44.5 & 50.0 & 52.8 \\
gas & 33.6 & 40.9 & 47.5 & 50.3
\end{tabular}

model and the Ewald summation. ${ }^{13}$ In both cases the densities used are lower than experiment (3.3\% and $4.2 \%$, respectively).

B. Molecular Conformation. Distributions of the dihedral angle $\phi$ associated with torsional motions about the $\mathrm{C}-\mathrm{O}$ bond $(S(\phi))$ were obtained during the MD runs. For the sake of comparison, $S(\phi)$ distributions corresponding to ideal gases (Boltzmann distributions) at the same temperatures that the simulated liquids were calculated. For all temperatures, $S(\phi)$ shows a central peak and two mirror-image peaks (Figure 1). The symmetry of the $S(\phi)$ peaks indicates that the conformational equilibrium was reached even at the lowest temperature. The central maximum corresponds to trans conformers, whereas the other two maxima correspond to gauche conformers. The central maximum for liquid is lower than for gas, whereas for the other two maxima the differences are in the opposite direction. The biggest differences are for the lowest temperature and, at $T=348 \mathrm{~K}$, the $S(\phi)$ functions for liquid and for gas are quite close.

The relative populations of conformers were calculated by integrating $S(\phi)$ between $120^{\circ}$ and $240^{\circ}$ (trans) and between $0^{\circ}$ and $120^{\circ}$ and $240^{\circ}$ and $360^{\circ}$ (gauche). At low $T$, the percentage of gauche conformers is lower than that of trans conformers while when $T$ increases the proportion tends to be reversed (Table 3). In all cases the percentage of gauche conformers is higher for liquid than for gas. This was also observed by Jorgensen, ${ }^{2}$ although he considered only a temperature $(298 \mathrm{~K})$ for which the differences between liquid and gas are rather small. Jorgensen associated this finding with a small steric preference of gauche molecules for hydrogen bonding due to the fact that for the gauche conformer the "lone-pair" region of $\mathrm{O}$ atoms is 
less encumbered by the alkyl chain than for the trans conformers. Results in this work support this interpretation since the differences between the percentages for gas and liquid become more important when the system has a higher degree of hydrogen bonding (see section III.E).

C. Comparison of MD Results with X-ray and Neutron Diffraction Data. Experimental information on the structure of liquids is obtained from X-ray or neutron-scattering measurements. Results obtained by these two methods are, in general, complementary. For example, in the case of ethanol, the X-ray patterns are dominated by scattering from the $\mathrm{C}$ and $\mathrm{O}$ atoms so that important information on $\mathrm{H}$-bonding is not directly accessible by this method. Neutron-scattering results of deuterated ethanol, however, have an important contribution from deuterium atoms. Ethanol molecules have 6 nonequivalent atoms and therefore 21 independent partial structure factors which, in practice, cannot be separated by isotopic substitution. Thus, the interpretation of diffraction results is difficult, and MD predictions based on realistic microscopic models are essential in order to complete the information on the structure of molecular systems. In this section, the structure of ethanol obtained from our MD simulations at $298 \mathrm{~K}$ is compared with $\mathrm{X}$-ray ${ }^{18}$ and neutron-scattering ${ }^{19}$ data.

The intermolecular structure of ethanol resulting from X-ray measurements ${ }^{18}$ was expressed in terms of the distinct structure function $H_{\mathrm{d}}(k)$ defined by

$$
H_{\mathrm{d}}(k)=\left[\sum\left(2-\delta_{\alpha \beta}\right) f_{\alpha}(k) f_{\beta}(k) \hat{h}_{\alpha \beta}(k)\right]\left[\sum f_{\alpha}(k)\right]^{-2}
$$

where sums are over all the scattering sites in a molecule. $f_{\alpha^{-}}$ (k) is the X-ray scattering factor of site $\alpha$ and $\hat{h}_{\alpha \beta}(k)$ is defined as the Fourier transformation of the corresponding site-site correlation function:

$$
\hat{h}_{\alpha \beta}(k)=4 \pi \rho_{\mathrm{s}} \int_{0}^{\infty} r h_{\alpha \beta}(r) \frac{\sin k r}{k} \mathrm{~d} r
$$

where $\rho_{\mathrm{s}}$ is the site number density and $h_{\alpha \beta}(r)=g_{\alpha \beta}(r)-1$. A weighted sum of the site-site correlation functions of distinct molecules is given by

$$
G_{\mathrm{d}}(r)=1+\left(2 \pi^{2} \rho_{\mathrm{s}} r\right)^{-1} \int_{0}^{\infty} k H_{\mathrm{d}}(k) \sin (k r) \mathrm{d} k
$$

Narten and Habenschuss ${ }^{18}$ reported $k H_{\mathrm{d}}(k)$ results for liquid ethanol at $293 \mathrm{~K}$. They treated the molecules as composed of three sites, i.e., $\mathrm{Me}_{\mathrm{O}}, \mathrm{Me}_{\mathrm{C}}$, and $\mathrm{O}$ (the contributions of the $\mathrm{H}$ atoms to the X-ray pattern are negligible). We calculated the MD three-site $H_{\mathrm{d}}(k)$ function from the corresponding $g(r)$ 's and the $f_{\alpha}(k)$ scattering factors given in Table 1 of ref 20 . The resulting $k H_{\mathrm{d}}(k)$ as well as the six partial contributions to this function are shown in Figure 2. Although all the partials contribute to the first peak of $k H_{\mathrm{d}}(k)$ at $1.7 \AA^{-1}$, the most important contribution corresponds to $\mathrm{Me}_{\mathrm{O}}-\mathrm{Me}_{\mathrm{O}}$ and to those involving the $\mathrm{Me}_{\mathrm{C}}$ site. At higher $k$ values, $k H_{\mathrm{d}}(k)$ is the result of the three pair correlations including O. Oscillations of $k H_{\mathrm{d}^{-}}$ (k) for $k$ higher than $8 \AA^{-1}$ practically coincide with the $\mathrm{O}-\mathrm{O}$ contribution. $k H_{\mathrm{d}}(k)$ obtained by $\mathrm{MD}$ is in agreement with $\mathrm{X}$-ray data and show only small discrepancies in the height of the first $k H_{\mathrm{d}}(k)$ peak and in the $2.5-4 \AA^{-1}$ region. The most important trend of the $G_{\mathrm{d}}(r)-1$ function (Figure 2) is the peak at $2.8 \AA$. We have verified that it must be associated with the $g_{\mathrm{OO}}(r)$ distribution function since the other contributions do not have any influence on this peak. We emphasize the good accord between the peak obtained by MD simulation and X-ray diffraction. This is consistent with the agreement between $k H_{\mathrm{d}^{-}}$ (k) from MD and experiments at high $k$ values.
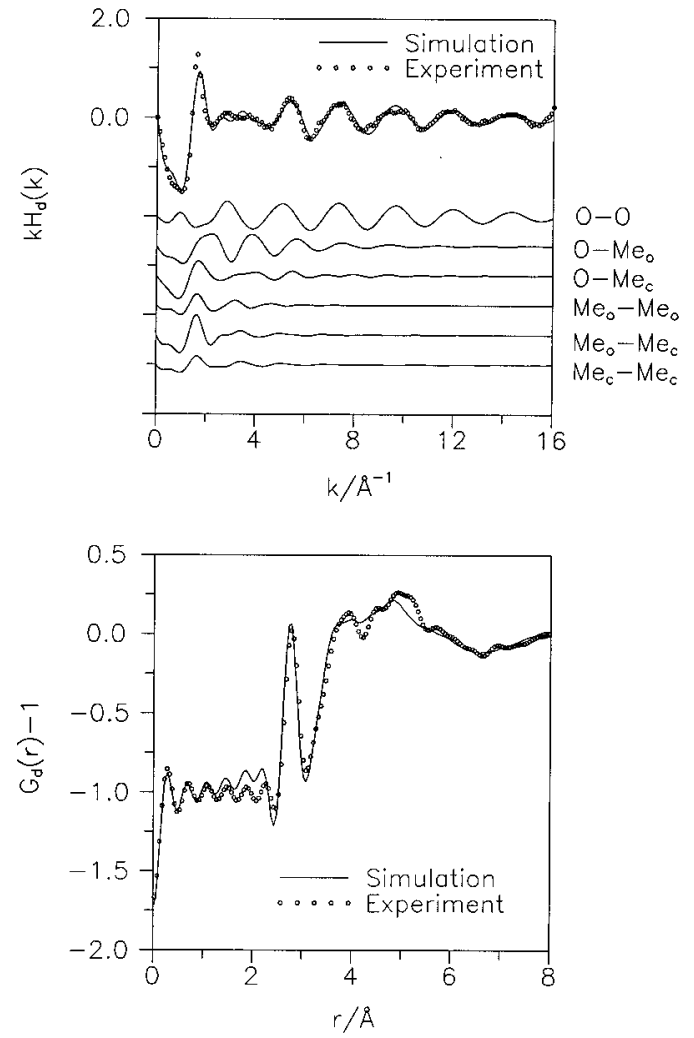

Figure 2. Intermolecular structure resulting from X-ray measurements compared with MD findings. The partial contributions to $k H_{\mathrm{d}}(k)$ obtained by MD are also represented (the origin of these curves is vertically displaced).

Information on the intermolecular structure of molecular liquids from neutron-scattering experiments is usually given through the $D_{\mathrm{M}}(k)$ function. ${ }^{21} d_{\mathrm{L}}(r)$ is defined as the Fourier inversion of $k D_{\mathrm{M}}(k)$

$$
d_{\mathrm{L}}(r)=(2 / \pi) \int_{0}^{\infty} k D_{\mathrm{M}}(k) \sin (k r) \mathrm{d} k
$$

This function is related to the total distribution function $g_{\mathrm{L}}(r)$ by

$$
d_{\mathrm{L}}(r)=4 \pi r \rho_{\mathrm{m}}\left(g_{\mathrm{L}}(r)-1\right)
$$

where $\rho_{\mathrm{m}}$ is the molecular number density. $g_{\mathrm{L}}(r)$ is a weighted sum of the atom-atom intermolecular radial distribution functions $g_{\alpha \beta}(r):^{21}$

$$
\begin{aligned}
& g_{\mathrm{L}}(r)= \\
& \quad\left[\sum\left(2-\delta_{\alpha \beta}\right) c_{\alpha} c_{\beta} b_{\alpha} b_{\beta} g_{\alpha \beta}(r)\right]\left[\sum\left(2-\delta_{\alpha \beta}\right) c_{\alpha} c_{\beta} b_{\alpha} b_{\beta}\right]^{-1}
\end{aligned}
$$

where sums run over all atoms of the molecule. $c_{\alpha}$ and $b_{\alpha}$ are the fractional concentration $\left(\sum c_{\alpha}=1\right)$ and the scattering length of species $\alpha$, respectively. It should be noted that neutronscattering experiments were carried out on deuterated ethanol. ${ }^{19}$ Thus, we use the appropriate scattering length of deuterium in the calculation of $g_{\mathrm{L}}(r)$.

Neutron-scattering data cannot be directly compared with our MD results since the $\mathrm{H}$ atoms of the methyl groups were not explicitly considered in our simulations, whereas they make a significant contribution to the neutron-scattering patterns. For this reason we performed complementary MD runs at $298 \mathrm{~K}$ which explicitly include the positions of the $\mathrm{H}$ atoms in the methyl groups but neglect the interactions of these $\mathrm{H}$ atoms. Methyl groups were held rigid according to the following conditions: ${ }^{22}$ (1) The $\mathrm{CH}$ bond length and the $\angle \mathrm{HCC}$ bond angle 

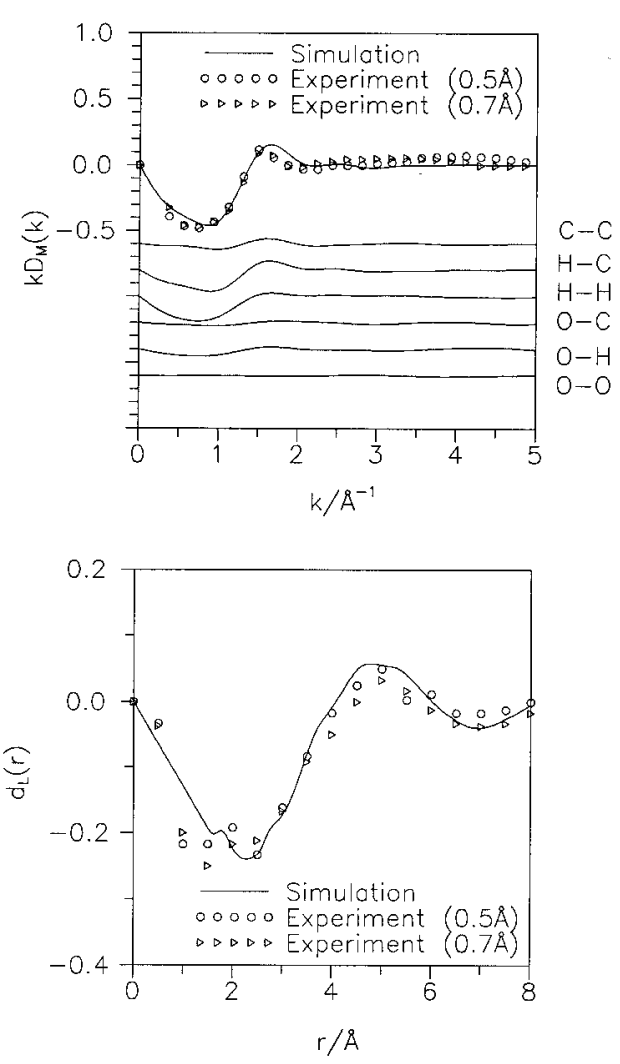

Figure 3. Intermolecular structure resulting from neutron scattering measurements for two incident wavelengths compared with MD findings. The contributions of different atom-atom correlations to $k D_{\mathrm{M}^{-}}$ $(k)$ are also plotted, but atoms of the same species in different groups are not distinguished (the origin of these curves is vertically displaced).

were kept at $1.0936 \AA$ and $110.297^{\circ}$, respectively. (2) Two conformations of the $\mathrm{CH}_{3}$ group were considered. If $\phi_{i}$ defines the angle between the $\mathrm{H}_{i} \mathrm{CC}$ and the $\mathrm{CCO}$ planes, the eclipsed conformation is defined by $\phi_{i}=0^{\circ}, 120^{\circ}$, and $240^{\circ}$ and the staggered conformation by $\phi_{i}=-60^{\circ},+60^{\circ}$, and $180^{\circ}$. For the $\mathrm{CH}_{2}$ group, $\phi_{i}=120^{\circ}$ and $240^{\circ}$. According to these constraints $\angle \mathrm{HCH}=108.633^{\circ}$ in all cases.

We calculated $d_{\mathrm{L}}(r)$ and $k D_{\mathrm{M}}(k)$ from the MD partial $g_{\alpha \beta}(r)$ functions by using eq 5-9. Accurate neutron-scattering data are extremely difficult to obtain for liquid ethanol. This results in large uncertainties which are shown by the differences between the two sets of experimental results at two values of the wavenumber ${ }^{19}$ (see Figure 3). The contributions to $k D_{\mathrm{M}}(k)$ for the different simulated partials are also shown in Figure 3. For the sake of simplicity contributions of atoms of the same species but in different groups of the ethanol molecule have not been plotted separately. The most significant trends in $k D_{\mathrm{M}^{-}}$ (k) are a broad minimum around $0.85 \AA^{-1}$ and a maximum around $1.7 \AA^{-1}$ which, on comparing with the X-ray diffraction data, should be associated with the correlations of $\mathrm{H}$ atoms. There are difficulties, however, for obtaining direct information on the $\mathrm{H}$ atom in the $\mathrm{OH}$ group as the most important $\mathrm{H}$ contribution to the diffraction pattern comes from the five $\mathrm{H}$ atoms in the methyl groups. Since the results obtained by either assuming the eclipsed or the staggered conformations are very close, we suggest that there is a need for more detailed simulations which include the intramolecular rotations of the $\mathrm{H}$ atom in the methyl group around the $\mathrm{C}-\mathrm{C}$ bond, as well as much more accurate experimental information to decide on the precise molecular conformation of liquid ethanol.

D. Intermolecular Structure. The structure of liquids is ordinarily expressed in terms of the partial radial distribution functions $g_{\alpha \beta}(r)$. The most structured and interesting $g_{\alpha \beta}(r)$
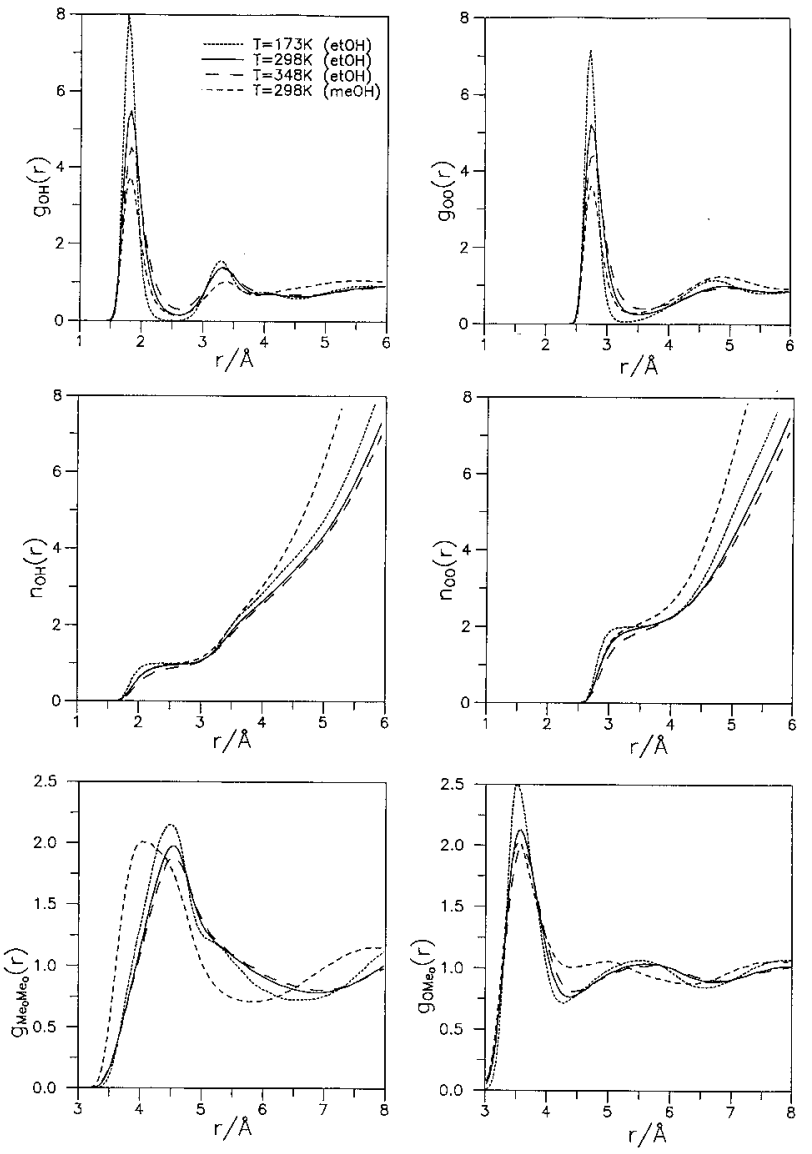

Figure 4. Partial radial distribution functions and coordination numbers for ethanol at three different temperatures and methanol at room temperature.

functions for liquid ethanol correspond to the $\mathrm{O}-\mathrm{H}, \mathrm{O}-\mathrm{O}$, $\mathrm{Me}_{\mathrm{O}}-\mathrm{Me}_{\mathrm{O}}$, and $\mathrm{O}-\mathrm{Me}_{\mathrm{O}}$ pairs. Our results at three different temperatures are shown in Figure 4. For the sake of clarity, the $g_{\alpha \beta}(r)$ functions at $T=223 \mathrm{~K}$, which are simply intermediate between those at 173 and $298 \mathrm{~K}$, are not shown. The coordination numbers $n_{\alpha \beta}(r)$ for the $\mathrm{O}-\mathrm{H}$ and $\mathrm{O}-\mathrm{O}$ pairs calculated by integration of the corresponding $g_{\alpha \beta}(r)$ functions are also plotted in Figure 4.

The $g_{\alpha \beta}(r)$ functions at $298 \mathrm{~K}$ are in agreement with those from earlier computer simulations. ${ }^{2,13}$ As expected, the liquid becomes more structured when $T$ decreases. The changes of temperatures do not affect significantly the positions of the $g_{\alpha \beta^{-}}$ ( $r$ ) maxima and minima but only their height and depth. Since the first minima of the $g_{\alpha \beta}(r)$ 's for $\mathrm{O}-\mathrm{H}$ and $\mathrm{O}-\mathrm{O}$ at $T=173$ $\mathrm{K}$ and $T=223 \mathrm{~K}$ are very close to 0 , the corresponding first coordination shells are very well defined, and the $n_{\alpha \beta}(r)$ functions for $\mathrm{O}-\mathrm{H}$ and $\mathrm{O}-\mathrm{O}$ show a clear plateau with $n_{\alpha \beta}(r)$ equal to 1 and 2, respectively. At higher temperatures, the $n_{\alpha \beta^{-}}$$(r)$ functions do not show horizontal plateaus in the regions of the $g_{\alpha \beta}(r)$ minima and the $n_{\alpha \beta}(r)$ values are slightly lower than either 1 or 2 .

The $g_{\alpha \beta}(r)$ functions for liquid methanol at $298 \mathrm{~K}$ obtained in earlier MD studies ${ }^{9,11}$ using the same OPLS potential model are compared with those for ethanol in Figure 4. As a general trend, the $g_{\alpha \beta}(r)$ functions for ethanol show higher maxima located at the same positions than those for methanol. The largest differences are for the $\mathrm{Me}_{\mathrm{O}}-\mathrm{Me}_{\mathrm{O}}$ pair. For the other $g_{\alpha \beta}(r)$ functions (including those not represented in Figure 4) the positions of the first maxima for ethanol and methanol are the same and small discrepancies can be observed only beyond the first peak. In our view, these results strongly suggest that the structure of both liquids is similar and predominantly 

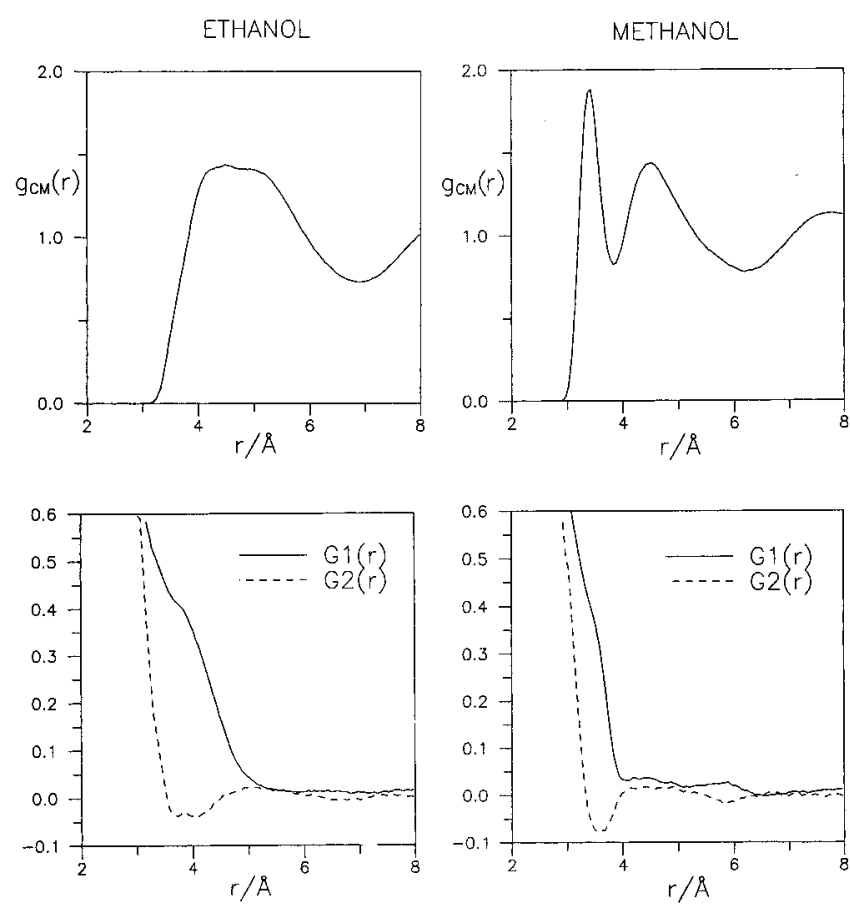

Figure 5. Radial distribution functions of the molecular center of mass and orientational correlation functions for ethanol and methanol at 298 K.

governed by chains of H-bonded molecules (see section III.F). Hence, the methyl group which distinguishes ethanol and methanol has little influence on the characteristics of the H-bonded chains. However, the volume occupied by this additional methyl group has a noticeable influence on the height of the peaks of the $g_{\alpha \beta}(r)$ functions. Consequently, at the same temperature $(298 \mathrm{~K})$, ethanol is more structured and methanol, although the molecular density of the former $\left(\rho_{\mathrm{m}}=1.03 \times 10^{-2}\right.$ molecule $\left.\AA^{-3}\right)$ is lower than that of the latter $\left(\rho_{\mathrm{m}}=1.48 \times\right.$ $10^{-2}$ molecule $\AA^{-3}$ ).

Orientational order in molecular liquids may be analyzed in terms of the following correlation functions: ${ }^{23}$

$$
G_{l}(r)=\left\langle P_{l}[\cos \vartheta(r)]\right\rangle
$$

where $P_{l}$ is the $l$ th Legendre polynomial and $\vartheta(r)$ is the angle between the dipole moments of two molecules whose centers of mass are located at a distance $r$. We calculated $G_{1}(r)$ and $G_{2}(r)$ and results for $T=298 \mathrm{~K}$ are shown in Figure 5. We have not found significant differences in the system at different temperatures. The resulting $G_{1}(r)$ and $G_{2}(r)$ functions show that orientational correlations decrease very fast when distance increases. At short distances $G_{1}(r)$ is positive, which indicates that the dipole moments of close molecules are parallel. These findings are similar to those for methanol (Figure 5). Actually methanol and ethanol results cannot be directly compared. Although in our molecular model the $\mathrm{Me}_{\mathrm{C}}$ group of ethanol has no electric charge, the mass of this group cannot be neglected. Thus, distances among the centers of mass for the two systems are not equivalent. This is clearly reflected by the remarkable differences between the $g_{\mathrm{CM}}(r)$ functions (Figure 5) although the atom-atom structures of the two liquids are quite similar.

E. Hydrogen Bonding. The criterion for deciding when two neighboring molecules are H-bonded is somewhat arbitrary. However, in the case of methanol the choice of a criterion is less crucial than for other liquids like water and both energetic and geometric criteria lead to very similar results. ${ }^{4}$ For the sake of simplicity we used a geometrical criterion. Since the positions of the first minima of $g_{\mathrm{OO}}(r)$ and $g_{\mathrm{OH}}(r)$ functions at
TABLE 4: Percentages of Molecules with $\boldsymbol{n}$ H-Bonds and Mean Number of H-Bonds per Molecule $\left(\left\langle n_{\mathrm{HB}}\right\rangle\right)$

\begin{tabular}{llll}
\hline & \multicolumn{3}{c}{$T(\mathrm{~K})$} \\
\cline { 2 - 4 }$n$ & 223 & 298 & 348 \\
\hline 0 & $<1$ & 1 & 4 \\
1 & 5 & 14 & 25 \\
2 & 92 & 80 & 66 \\
3 & 3 & 5 & 5 \\
$\left\langle n_{\mathrm{HB}}\right\rangle$ & 2.0 & 1.9 & 1.7
\end{tabular}

$298 \mathrm{~K}$ are very close to those for methanol we have chosen the same H-bond definition and cutoff distances as in earlier H-bonding studies of methanol.,6,11 We also employed the same H-bond definition for runs at different temperatures. Although the positions of the $g_{\alpha \beta}(r)$ minima show slight shifts with temperature (Figure 4), the changes are rather small, especially for $g_{\mathrm{OH}}(r)$ which is the most crucial cutoff. A pair of ethanol molecules have been considered H-bonded if three conditions are fulfilled, namely, the $\mathrm{O} \cdots \mathrm{O}$ distance is smaller than $3.5 \AA$, the $\mathrm{O} \cdots \mathrm{H}$ distance is smaller than $2.6 \AA$ and the $\mathrm{HO} \cdots \mathrm{O}$ angle is smaller than $30^{\circ}$. We define the $\mathrm{H}$-bonding state of an ethanol molecule in a given configuration according to the number of $\mathrm{H}$-bonds that it has. We determined the number of H-bonds per molecule independently on whether its $\mathrm{O}$ atom was an acceptor or a donor. We did not find significant differences in the calculated properties when this distinction was taken into account.

The averaged fractions of molecules in different H-bonding states were calculated from the MD configurations and the results are summarized in Table 4 . Given the difficulties in carrying out reliable statistical averages, we cannot give results at $173 \mathrm{~K}$. As the $\mathrm{H}$-bond lifetimes at this temperature are very large $\left(\cong 10^{2}\right.$ ps for liquid methanol ${ }^{5,6}$ ), the H-bonded structure did not show significant changes throughout our MD runs. Findings at $298 \mathrm{~K}$ are in agreement with those obtained by Jorgensen $^{2}$ using an energetic criterion to define the H-bonds. Changes of H-bonding with $T$ are consistent with those found for liquid methanol using different potentials and $\mathrm{H}$-bond definitions. ${ }^{4-6,11}$

According to the $\left\langle n_{\mathrm{HB}}\right\rangle$ results, shown in Table 4, the majority of molecules in the simulated systems have two H-bonds. This suggests that the structure of liquid ethanol at different temperatures is basically constituted by linear chains of H-bonded molecules. Molecules with three H-bonds $(n=3)$ should be associated with branching. The fraction of molecules in this $\mathrm{H}$-bonding state is small at all temperatures while fractions for $n=2$ and $n=1$, which correspond to molecules in the middle and at the end of the chain, respectively, are markedly dependent on $T$. It follows from our findings that chains become longer as $T$ decreases, whereas the number of branches is kept constant or even decreases. The number of non-H-bonded molecules increases with $T$ but even at $348 \mathrm{~K}$ is rather low.

F. Hydrogen-Bonded Chains. A careful analysis of different configurations has corroborated the existence of winding chains with several branches analogous to those observed in earlier simulation studies of other liquid alcohols. ${ }^{2,4,5}$ To study in detail their characteristics, we identified the H-bonded chains existing in 50 configurations at each temperature. These configurations were saved during the MD simulations every 200 time steps. We considered the possibility that the chains cross the wall of the central box by taking into account the images given by the periodic boundary conditions. However, in this case, the corresponding particles into the box were excluded, so that the total number of molecules in the different chains of 

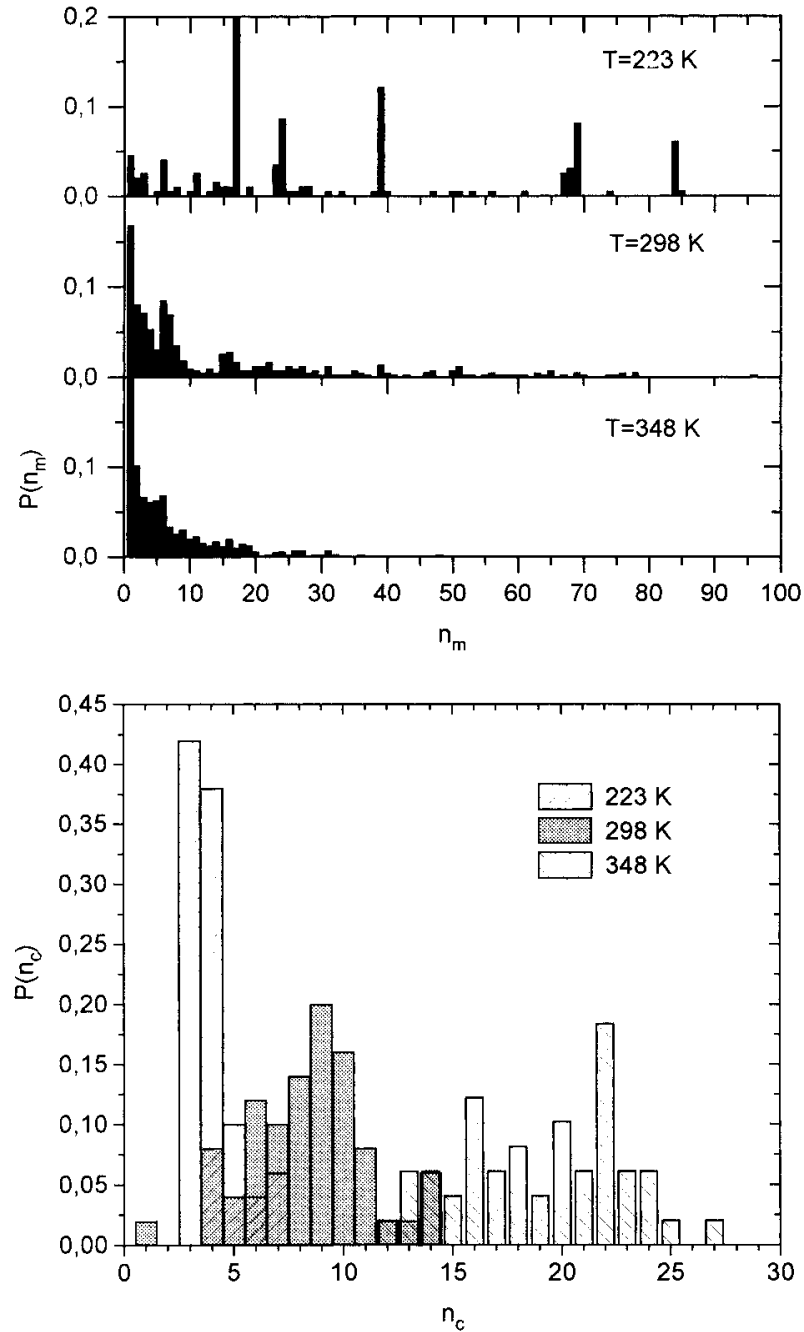

Figure 6. Probability that the number of monomers of a chain is $n_{\mathrm{m}}$ (top) and that the number of chains per configuration in $n_{\mathrm{c}}$ (bottom).

each configuration was 125 . Probabilities of finding both chains with $n_{\mathrm{m}}$ monomers $\left(P\left(n_{\mathrm{m}}\right)\right)$ and $n_{\mathrm{c}}$ chains per configuration $\left(P\left(n_{\mathrm{c}}\right)\right)$ were calculated, and the results are shown in Figure 6. These probabilities were obtained considering all the molecules of a chain independently of whether they are in different branches.

At the highest $T$, there are many chains (between 12 and 25) with a rather small number of monomers. Moreover, $P\left(n_{\mathrm{m}}\right)$ at this temperature shows an exponential decrease which is characteristic of randomly bonded molecules. At $T=223 \mathrm{~K}$, however, the number of chains is small (between 3 and 7), and there is a significant probability of finding long chains. In this case, $P\left(n_{\mathrm{m}}\right)$ is not exponential. $P\left(n_{\mathrm{c}}\right)$ and $P\left(n_{\mathrm{m}}\right)$ at $T=298 \mathrm{~K}$ are intermediate between those at $T=348 \mathrm{~K}$ and $T=223 \mathrm{~K}$ and $P\left(n_{\mathrm{m}}\right)$ shows an approximately exponential decrease. We could not obtain reliable results at $173 \mathrm{~K}$ for the reasons given in the preceding section.

Sarkar and Joarder ${ }^{24}$ suggested that hexamer closed chains of ethanol monomers could be a majority at room temperature. However, the agreement between experimental and theoretical results assuming this chain model is not much better than that obtained for other models such as a U-shaped chain or a tetramer closed chain. This shows the difficulties for obtaining detailed information on the intermolecular structure from experimental data. A careful examination of our saved MD configurations has shown that there are very few closed chains. Moreover, we have not observed any predominant shape of the chains.
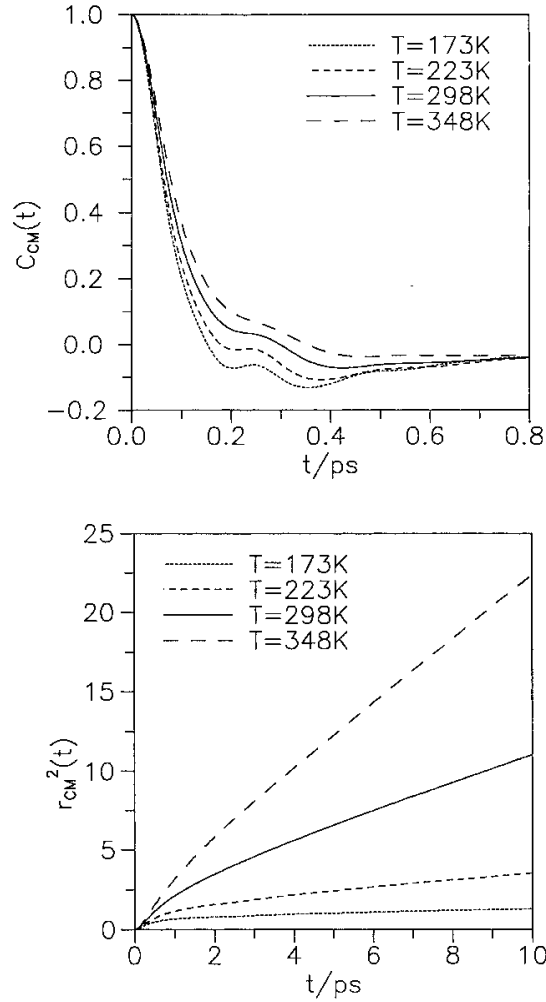

Figure 7. Velocity autocorrelation functions (top) and mean-square displacements (bottom) of the center of mass of ethanol molecules at different temperatures.

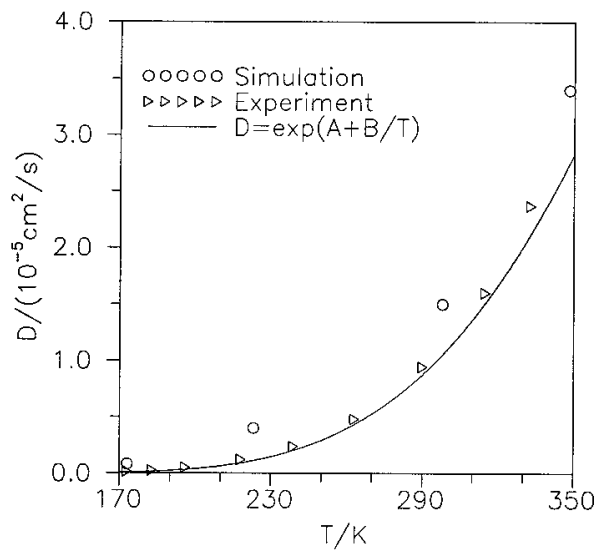

Figure 8. Self-diffusion coefficients from this work and from NMR experiments. Parameters corresponding to the continuous line were obtained from experimental data. ${ }^{26}$

\section{Dynamical Properties}

A. Self-Diffusion Coefficients. The self-diffusion coefficients $(D)$ were calculated from the long-time slope of the mean-square displacements of the center of mass of ethanol molecules (Figure 7) according to the Einstein relation. The $D$ coefficients show a marked decrease with temperature, and they are very small at $173 \mathrm{~K}$. This decrease is consistent with the formation of longer $\mathrm{H}$-bonded chains at low temperatures. Our $D$ coefficients are compared with those from NMR experiments $^{25}$ at $P=0.1 \mathrm{MPa}$ (which correspond to the densities in our MD simulations) in Figure 8 . The equation $D=\exp (A+$ $B T^{-1}$ ) with values of parameters determined from experimental data between 285 and $320 \mathrm{~K}^{26}$ is also plotted in Figure 8 . Changes of $D$ with temperature are acceptably reproduced by our simulation results although the MD coefficients are somewhat larger than experimental values. 

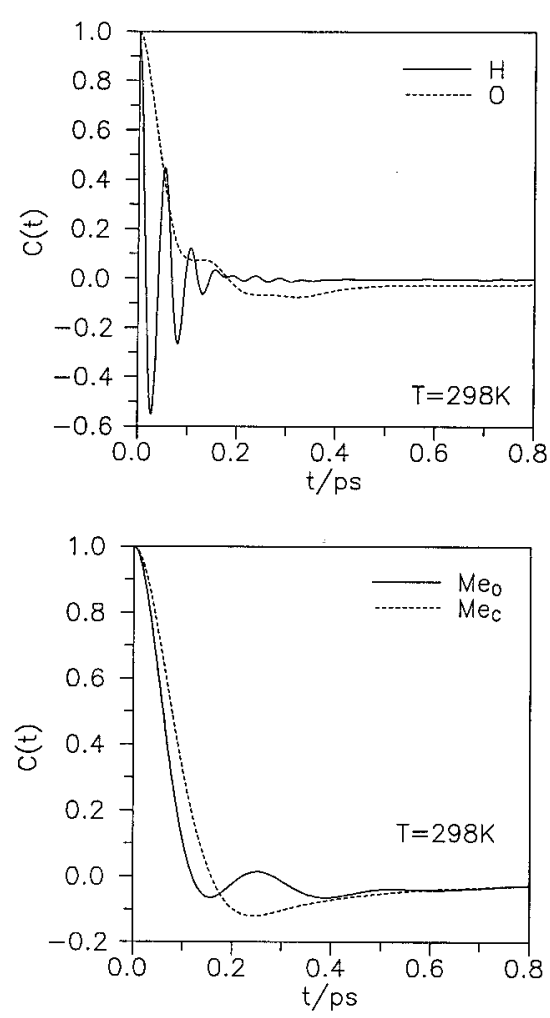

Figure 9. Velocity autocorrelation functions of the $\mathrm{H}$ and $\mathrm{O}$ atoms (top) and the methyl groups (bottom) of ethanol molecules.

B. Velocity Autocorrelation Functions and Spectral Densities. The normalized velocity autocorrelation functions $(C(t))$ corresponding to the molecular center of mass (CM), the $O$ atom, the $\mathrm{H}$ atom of the hydroxyl group as well as to the $\mathrm{Me}_{\mathrm{O}}$ and $\mathrm{Me}_{\mathrm{C}}$ groups were determined during the MD simulations. The $C_{\mathrm{CM}}(t)$ functions show two submaxima which are more marked at lower $T$ (Figure 7). The $C(t)$ functions for atoms and atomic groups in the ethanol molecules corresponding to the run at $298 \mathrm{~K}$ are plotted in Figure 9. The shapes of these functions at the other temperatures are similar to those at 298 $\mathrm{K}$. The most significant change is that oscillations in $C_{\mathrm{O}}(t)$ and $C_{\mathrm{H}}(t)$ become more marked and the $C(t)$ functions for methyl groups reach more negative values as $T$ decreases. $C_{\mathrm{Me}_{\mathrm{c}}}(t)$ shows a minimum and has the characteristic shape of the velocity autocorrelation function of dense monoatomic liquids. Thus, the submaxima in $C_{\mathrm{CM}}(t)$ should be associated to the $\mathrm{O}$ and $\mathrm{Me}_{\mathrm{O}}$ motions which are more affected by the intramolecular bonds. Because of their small mass the contribution of $\mathrm{H}$ atoms to $C_{\mathrm{CM}}(t)$ is rather small. $C_{\mathrm{H}}(t)$ reflects the fact that motions of $\mathrm{H}$ atoms are predominantly oscillatory. However, these oscillations cannot be attributed to the high-frequency vibrational motions along the $\mathrm{O}-\mathrm{H}$ bond (stretching) since this intramolecular degree of freedom is not considered in the potential model used in this work.

Atomic motions in liquids are frequently analyzed through the spectral densities $S(\omega)$ which are defined as the Fourier transforms of the corresponding $C(t)$ functions. $S(\omega)$ provides information on the relative number of modes for a frequency $\omega$, and it is related with the experimental infrared power spectra. ${ }^{27}$ Our MD results at $298 \mathrm{~K}$ are shown in Figure 10. $S_{\mathrm{O}}(\omega), S_{\mathrm{Me}_{\mathrm{O}}}(\omega)$, and $S_{\mathrm{Me}_{\mathrm{C}}}(\omega)$ show a low-frequency peak which is characteristic of the spectral densities for dense liquids. These maxima and the nonvanishing $S(0)$ values indicate that molecular motions may be considered as a combination of both the temporary vibrations of a tagged molecule inside the cage formed by their neighbors and the diffusive translational motions typical of liquids. $S_{\mathrm{O}}(\omega)$ and $S_{\mathrm{Meo}}(\omega)$ show also a shoulder at

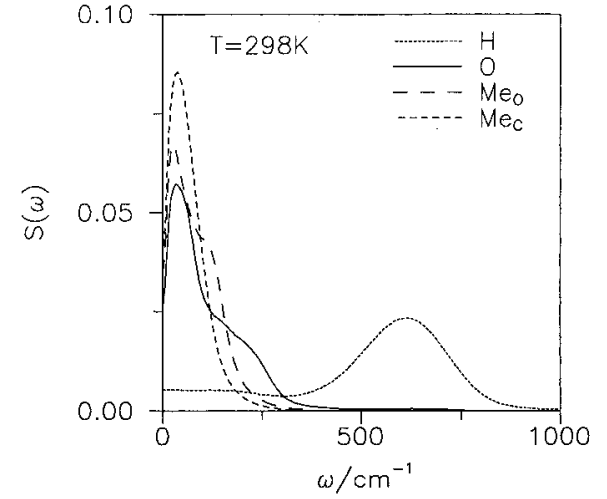

Figure 10. Spectral densities corresponding to $\mathrm{H}$ and $\mathrm{O}$ atoms and methyl groups.

higher frequencies whose origin will be discussed below. The small $S_{\mathrm{H}}(0)$ value and the $S_{\mathrm{H}}(\omega)$ band with a maximum at $\omega$ higher than $500 \mathrm{~cm}^{-1}$ corroborate our view that $\mathrm{H}$ motions are predominantly oscillatory as corresponds to the smaller mass of the $\mathrm{H}$ atoms. In liquid water, this band has been associated with librational modes. ${ }^{27}$

Intermolecular $\mathrm{H}$-bonds have a strong influence on the infrared and Raman spectra of liquids and, therefore, on the $S(\omega)$ functions. One of the advantages of MD is that it allow us to calculate separately the properties of molecules in different H-bonding states. We calculated the $C(t)$ functions for molecules with $0,1,2$, and $3 \mathrm{H}$-bonds during the MD simulation at $298 \mathrm{~K}$. We classified the molecules at the different initial configurations and it was assumed that their $\mathrm{H}$-bonding states did not change during the time required for the $C(t)$ function to go to zero. This procedure was already used to analyze the H-bond influence on $S(\omega)$ for other liquids. ${ }^{11,28}$ The $S(\omega)$ spectra corresponding to molecules with two H-bonds (Figure 11) are very similar to those for the full system (Figure 10) because the most part of molecules are in the $n=2$ state whereas the number of molecules with $n=0$ and $n=3$ is rather small (see Table 4). Hence, the contribution of the latter to the total spectra will be very weak and hardly detectable by experiment.

According to the findings shown in Figure 11, the lowfrequency maximum of $S_{\mathrm{O}}(\omega)$ is independent of the H-bonding state of molecules, whereas the shoulder around $200 \mathrm{~cm}^{-1}$ must be associated with the H-bonds. This shoulder does not appear for non-H-bonded molecules, and it is clearly shown by $S_{\mathrm{O}}(\omega)$ for molecules with two H-bonds. In the case of $n=3$ there is also a maximum in this region whereas for $n=1$ we can observe an incipient shoulder. Recent MD studies of $S_{\mathrm{O}}(\omega)$ for both liquid methanol ${ }^{11}$ and water ${ }^{28}$ have also shown the existence of similar $S_{\mathrm{O}}(\omega)$ shoulders which were associated with stretching intermolecular vibrations of molecules in the H-bonded chains. The first $S_{\mathrm{O}}(\omega)$ maximum attributed to the cage vibrations was also observed in liquid methanol and water at high densities. In the case of ethanol we have also analyzed $S_{\mathrm{Meo}}(\omega)$ for molecules in different H-bonding states. Results in Figure 11 indicate that features of the spectra around $100 \mathrm{~cm}^{-1}$ should be related to molecules forming two and three $\mathrm{H}$-bonds, whereas the first maximum has no relation with $\mathrm{H}$-bonds. These findings are consistent with the interpretation of the $S_{\mathrm{O}}(\omega)$ spectra and show that although the $\mathrm{Me}_{\mathrm{o}}$ group has no direct participation in the H-bonds, its motion is strongly dependent on the $\mathrm{H}$-bonding state of the molecule. On the other hand, the $\mathrm{Me}_{\mathrm{C}}$ group is not significantly affected by H-bonding and its motion show characteristics close to those of nonassociated liquids.

In the case of $\mathrm{H}$, it is useful to analyze the $S_{\mathrm{H}}(\omega)$ spectra for $\mathrm{H}$-bonded and non-H-bonded atoms separately. Our results for 

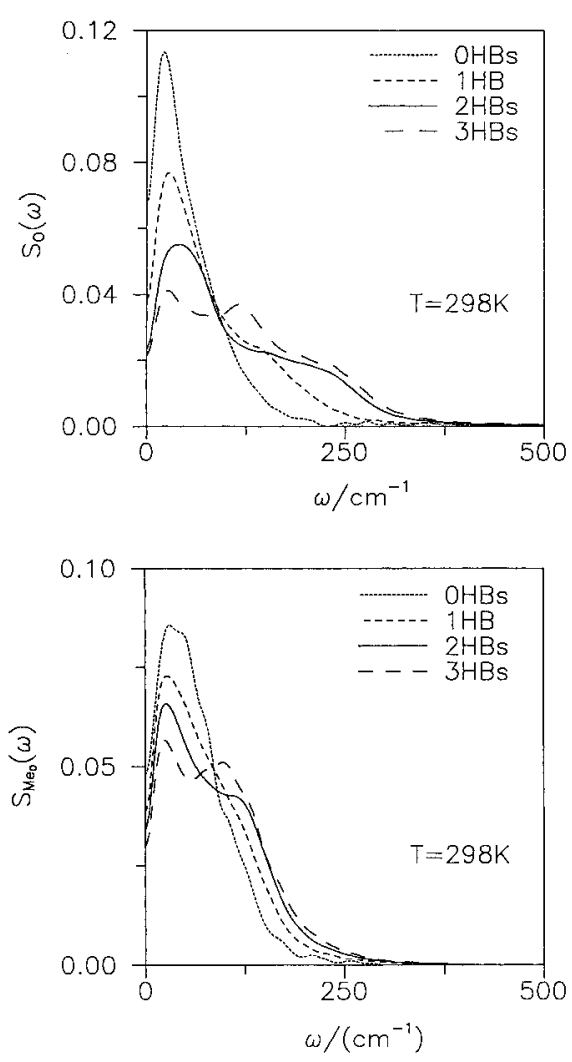

Figure 11. Spectral densities for the $\mathrm{O}$ atoms (top) and the $\mathrm{Me}_{\mathrm{o}}$ groups (bottom) in ethanol molecules at different H-bonding states.

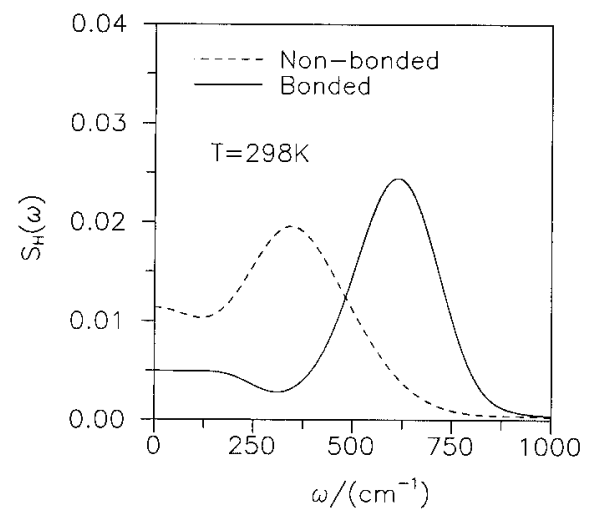

Figure 12. Spectral densities corresponding to H-bonded and non H-bonded hydrogen atoms.

ethanol at $298 \mathrm{~K}$ (Figure 12) show that H-bonds produce a marked shift $\left(\Delta \omega \cong 260 \mathrm{~cm}^{-1}\right.$ ) of the spectral band toward the higher frequency region. A similar shift was observed in the case of the librational frequency band for liquid water. ${ }^{28}$

C. Reorientational Motions. Reorientational molecular motions in liquids are usually analyzed through the time correlation functions

$$
C_{l}(t)=\left\langle P_{l}(\cos \theta(t))\right\rangle
$$

where $P_{l}$ refers to the $l$ th Legendre polynomial and $\theta(t)$ is the angle through which a molecule-fixed vector rotates in a time $t$. We calculated $C_{1}(t)$ and $C_{2}(t)$ for both the electric dipole moment $(\vec{\mu})$ and the $\mathrm{O}-\mathrm{H}$ vector. $C_{1}(t)$ for $\vec{\mu}$ and $C_{2}(t)$ for $\mathrm{O}-\mathrm{H}$ are related to the dielectric and NMR relaxation measurements, respectively. At long times, the $C_{l}(t)$ functions display a Debye-like exponential behavior, whereas at short times, they show a faster nonexponential decay (Figure 13). This initial decay, more apparent for $\vec{\mu}$ than for $\mathrm{O}-\mathrm{H}$, has been related to
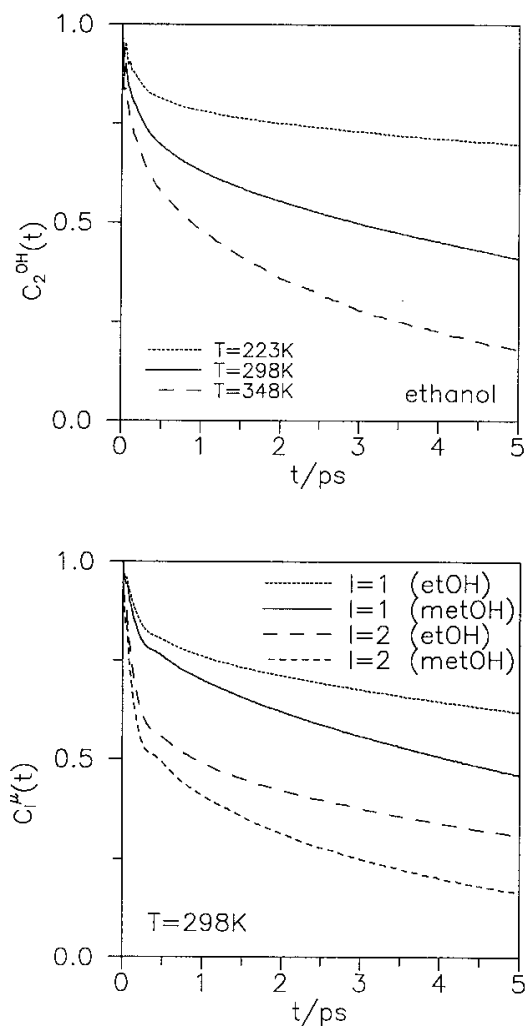

Figure 13. Reorientational time correlation functions for the $\mathrm{OH}$ direction (top) and the molecular dipole moment (bottom).

TABLE 5: Reorientational Correlation Times (in ps)

\begin{tabular}{|c|c|c|c|c|c|c|}
\hline & \multicolumn{6}{|c|}{$T(\mathrm{~K})$} \\
\hline & \multicolumn{2}{|c|}{223} & \multicolumn{2}{|c|}{298} & \multicolumn{2}{|c|}{348} \\
\hline & $\vec{\mu}$ & $\mathrm{O}-\mathrm{H}$ & $\vec{\mu}$ & $\mathrm{O}-\mathrm{H}$ & $\vec{\mu}$ & $\mathrm{O}-\mathrm{H}$ \\
\hline$\tau_{1}^{\mathrm{I}}$ & 150 & 175 & 20 & 23 & 6 & 7 \\
\hline$\tau_{1}^{\mathrm{E}}$ & 185 & 200 & 27 & 29 & 9 & 8 \\
\hline$\tau_{2}^{\mathrm{I}}$ & 45 & 55 & 6 & 7 & 2 & 2 \\
\hline$\tau_{2}^{\mathrm{E}}$ & 75 & 80 & 14 & 12 & 4 & 4 \\
\hline
\end{tabular}

the molecular librational motions in associated liquids. ${ }^{4}$ As expected, molecular reorientation of ethanol is slower than that for methanol and it becomes faster when $T$ increases (Figure 13).

To obtain a quantitative estimate of the reorientational correlation times, exponential functions were fitted to the longtime parts of the $C_{l}(t)$ functions

$$
C_{l}(t) \rightarrow \exp \left(-t / \tau_{l}^{\mathrm{E}}\right)
$$

Integral reorientational correlation times were also calculated:

$$
\tau_{l}^{\mathrm{I}}=\int_{0}^{\infty} C_{l}(t) \mathrm{d} t
$$

The exponential fit with $\tau_{l}^{\mathrm{E}}$ (eq 10) was used to compute the long-time parts of these integrals.

The results for $\tau_{l}^{\mathrm{E}}$ and $\tau_{l}^{\mathrm{I}}$ results are shown in Table 5 . Reorientational motions at $173 \mathrm{~K}$ are very slow, and the calculation of the corresponding correlation times would require to perform MD runs much longer than those done in this work. $\tau_{2}^{\mathrm{I}}$ values can be compared with data from NMR measurements (18 ps at $298 \mathrm{~K}$ and 133 ps at $223 \mathrm{~K}){ }^{29}$ Experimental values are higher than those from MD but the relation between $\tau_{2}^{\mathrm{I}}$ at the two temperatures is similar, i.e., 7.8 from MD and 7.4 from NMR experiments. Marked discrepancies were also observed 
between MD and NMR results for methanol at different temperatures. ${ }^{30}$ Since there are large uncertainties in the evaluation of $\tau_{2}^{\mathrm{I}}$ both from NMR experiments and MD simulations the differences between the two are between the error bars. The dielectric longitudinal (microscopic) correlation times (33 ps at $298 \mathrm{~K}$ and 294 ps at $223 \mathrm{~K}$ ) obtained from measurements of the macroscopic Debye relaxation times ${ }^{29}$ are also longer than the reorientational correlation times of the dipole moment resulting from our MD simulations.

\section{Concluding Remarks}

Results in this work show that there is, in general, good agreement between MD findings and available experimental information. Thermodynamic and structural data from experiments are in acceptable accordance with those from MD simulations although it should be pointed out that there are structural details, such as the $g_{\alpha \beta}(r)$ functions, which cannot be reliably checked with the actual neutron and X-ray data. In the case of dynamic properties, changes with temperature are reasonably reproduced but both translational and reorientational motions from MD are faster than those deduced from experimental findings. Despite these quantitative disagreements, our MD simulation results indicate that the OPLS potential is a simple and useful model for the intermolecular interactions in liquid ethanol which has enabled us to perform realistic simulations of the microscopic behavior of this liquid at different thermodynamic states.

As in the case of liquid methanol, both structural and dynamical properties of liquid ethanol are strongly influenced by intermolecular $\mathrm{H}$-bonds. Its structure is dominated by winding chains made up of a higher number of monomers at lower temperatures. We have corroborated that spectral densities, which are related with results of spectroscopic measurements, are strongly dependent on the H-bonding states of molecules.

Acknowledgment. We thank Dr. M. Silbert for useful comments and suggestions. L.S. gratefully acknowledges a FPI fellowship from Ministerio de Educación y Ciencia. This work has been supported by DGICYT through PB-93-0971-C03 Grant and by CIRIT through a SGR Grant.

\section{References and Notes} 335 .

(1) Ladanyi, B. M.; Skaf, M. S. Annu. Rev. Phys. Chem. 1993, 44,

(2) Jorgensen, W. L. J. Phys. Chem. 1986, 90, 1276.

(3) Pálinkás, G.; Hawlicka, E.; Heinzinger, K. J. Phys. Chem. 1987, 91, 4334.

(4) Haughney, M.; Ferrario, M.; McDonald, I. R. J. Phys. Chem. 1987, 91, 4934.

(5) Matsumoto, M.; Gubbins, K. E. J. Chem. Phys. 1990, 93, 1981.

(6) Alonso, J.; Bermejo, F. J.; García-Hernandez, M.; Martínez, J. L.; Howells, W. S. J. Mol. Struct. 1991, 250, 147.

(7) Casulleras, J.; Guàrdia, E. Mol. Simul. 1992, 8, 273. 8929.

(8) Skaf, M. S.; Fonseca, T.; Ladanyi, B. M. J. Chem. Phys. 1993, 98 ,

(9) Guàrdia, E.; Sesé, G.; Padró, J. A. J. Mol. Liq. 1994, 62, 1.

(10) Svishchev, I. M.; Kusalik, P. G. J. Chem. Phys. 1994, 100, 5165

(11) Martí, J.; Padró, J. A.; Guàrdia, E. J. Mol. Liq. 1995, 64, 1.

(12) Jorgensen, W. L. J. Am. Chem. Soc. 1981, 103, 345.

(13) van Leeuwen, M. E. Mol. Phys. 1996, 87, 87.

(14) Gao, J.; Habibollazadeh, D.; Shao, L. J. Phys. Chem. 1995, 99, 16460

(15) Caldwell, J. W.; Kollman, P. A. J. Phys. Chem. 1995, 99, 6208.

(16) See, for example, Perera, L.; Essman, U.; Berkowitz, M. L. J. Chem. Phys. 1995, 102, 450, and references therein.

(17) (a) Wilhoit, R. C.: Zwolinski, B. J. J. Phys. Chem. Ref. Data 1973 Suppl. 2. (b) Smith, B. D.; Srivastava, R. Thermodynamic Data for Pure Compounds; Elsevier: Amsterdam, 1986.

(18) Narten, A. H.; Habenschuss, A. J. Chem. Phys. 1984, 80, 3387.

(19) Montague, D. G.; Gibson, I. P.; Dore, J. C. Mol. Phys. 1982, 47, 1405.

(20) Narten, A. H. J. Chem. Phys. 1979, 70, 299.

(21) Dore, J. C. In Molecular Liquids; Reidel: Dordrecht, 1984; NATO ASI Series C, Vol. 135, p 383.

(22) Jorgensen, W. L. J. Am. Chem. Soc. 1981, 103, 341.

(23) Böhm, H. J.; McDonald, I. R.; Madden, P. A. Mol. Phys. 1983 49,347

(24) Sarkar, S.; Joarder, R. N. J. Chem. Phys. 1994, 100, 5118.

(25) Karger, N.; Verdag, T.; Lüdemann, H. D. J. Chem. Phys. 1990 93, 3437.

(26) Meckl, S.; Zeidler, M. D. Mol. Phys. 1988, 63, 85.

(27) Martí, J.; Guàrdia, E.; Padró, J. A. J. Chem. Phys. 1994, 101, 10883

(28) Martí, J.; Padró, J. A.; Guàrdia, E. J. Chem. Phys. 1996, 105, 639.

(29) Ludwig, R.; Zeidler, M. D.; Farrar, T. C. Z. Phys. Chem. 1995, $189,19$.

(30) Ludwig, R.; Rusbüldt, Ch.; Bopp, Ph. A.; Zeidler, M. D. Z. Naturforsch. 1995, 50a, 211. 